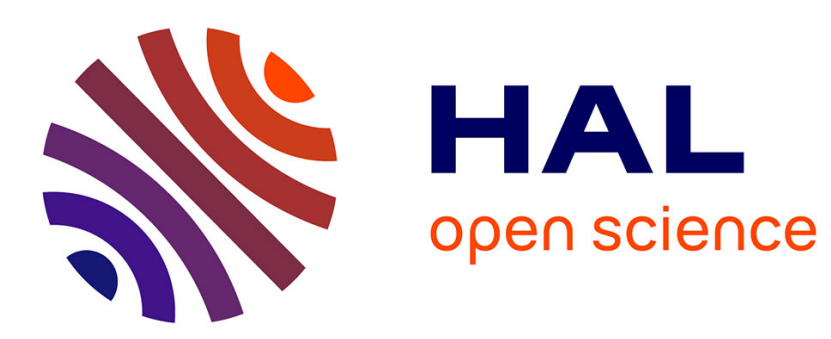

\title{
Optical properties of lattice/magnetic small polarons from DMFT
}

S. Ciuchi, E. Cappelluti, Simone Fratini

\section{To cite this version:}

S. Ciuchi, E. Cappelluti, Simone Fratini. Optical properties of lattice/magnetic small polarons from DMFT. Journal of Physics and Chemistry of Solids, 2008, 69 (9), pp.2164-2167. 10.1016/j.jpcs.2008.03.023 . hal-00551214

\section{HAL Id: hal-00551214 https://hal.science/hal-00551214}

Submitted on 3 Jan 2011

HAL is a multi-disciplinary open access archive for the deposit and dissemination of scientific research documents, whether they are published or not. The documents may come from teaching and research institutions in France or abroad, or from public or private research centers.
L'archive ouverte pluridisciplinaire HAL, est destinée au dépôt et à la diffusion de documents scientifiques de niveau recherche, publiés ou non, émanant des établissements d'enseignement et de recherche français ou étrangers, des laboratoires publics ou privés. 


\section{Author's Accepted Manuscript}

Optical properties of lattice/magnetic small polarons from DMFT

S. Ciuchi, E. Cappelluti, S. Fratini

PII:

S0022-3697(08)00095-4

DOI: doi:10.1016/j.jpcs.2008.03.023

Reference: PCS 5426

To appear in: $\quad$ Journal of Physics and

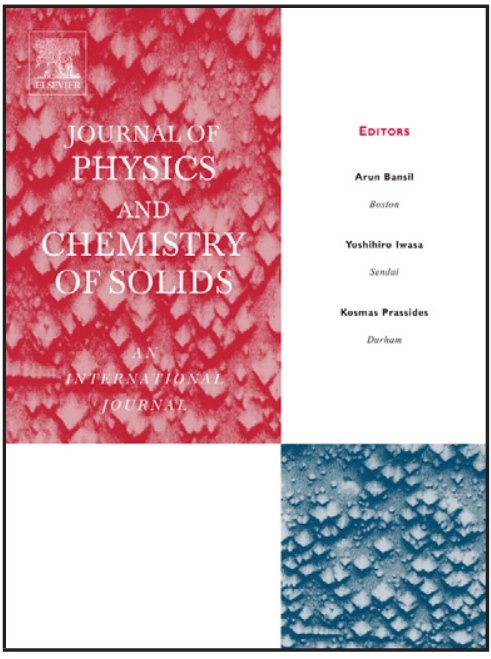

www.elsevier.com/locate/jpcs Chemistry of Solids

Cite this article as: S. Ciuchi, E. Cappelluti and S. Fratini, Optical properties of lattice/magnetic small polarons from DMFT, Journal of Physics and Chemistry of Solids (2008), doi:10.1016/j.jpcs.2008.03.023

This is a PDF file of an unedited manuscript that has been accepted for publication. As a service to our customers we are providing this early version of the manuscript. The manuscript will undergo copyediting, typesetting, and review of the resulting galley proof before it is published in its final citable form. Please note that during the production process errors may be discovered which could affect the content, and all legal disclaimers that apply to the journal pertain. 


\title{
Optical properties of lattice/magnetic small polarons from DMFT
}

\author{
S. Ciuchi ${ }^{1}$ E. Cappelluti ${ }^{2}$ S. Fratini ${ }^{3}$ \\ ${ }^{1}$ Istituto Nazionale di Fisica della Materia \& Dipartimento di Fisica Università dell'Aquila, Italy \\ via Vetoio, I-67010 Coppito-L'Aquila, Italy \\ ${ }^{3}$ SMC Research Center and ISC, INFM-CNR, v. dei Taurini 19, 00185 Rome, Italy, \\ Dipartimento di Fisica, Università "La Sapienza", P.le A. Moro 2, 00185 Rome, Italy \\ ${ }^{2}$ Institut Néel - CNRS \& Université Joseph Fourier, BP166, F-38042 Grenoble, France
}

\begin{abstract}
We calculate the optical conductivity of small polarons in the Holstein and Holstein- $t-J$ models, by applying the Dynamical Mean Field Theory. We show that the antiferromagnetic correlations tend to increase the region of the parameters where polaronic signatures are found in the optical spectra, and shift the polaronic absorption band to higher frequencies compared to the case of purely lattice polarons. On the other hand, the electron-lattice interaction is essential in order to have polaronic features in the optical absorption.
\end{abstract}

Key words: magnetic/lattice polarons, spin fluctuations, optical conductivity, cuprates. PACS: 71.10.Fd, 71.38.Ht, 78.20.Bh, 75.30.Ds.

\section{Introduction}

In systems with strong electron-phonon (e-ph) interactions, the motion of the carriers is significantly reduced by the formation of polarons. A polaron is a state in which the phonon and electron degrees of freedom are tightly entangled: the presence of an electron is associated to a finite lattice distortion, which in turn binds the electron, leading to the so-called self-trapping effect. Magnetic interactions, such as those acting on an hole moving in antiferromagnetic ( $\mathrm{AF}$ ) background, also tend to localize the charges. In this case the localizing effect is due to the energy cost that the moving hole has to pay as it modifies the local magnetic environment. In transition metal oxides, these two mechanisms often coexist, leading to an interesting interplay which will be the subject of the present study.

Typical signatures of polarons are seen in photoemission spectra[1] — where the lattice degrees of freedom can show up as characteristic multipeaked structures - and transport measurements - where a thermally activated behavior is often observed at room temperature[2,3]. Optical absorption measurements $[4,5]$ may also detect a polaronic band at a frequency related to the polaron binding energy, [6] as well as polaronic interband transitions in the range of the phonon frequencies [7]. Another less classical indication of polaronic formation comes from the analysis of lattice displacements associated to the excess charge as obtained by the distribution of distances between atoms[8]. 
In studies of polaron formation, the difficulty consists in describing the dressing of the moving electron by a cloud of phonons, in a complex state that can coherently move as a quasiparticle. [9] Here we use Dynamical Mean Field Theory (DMFT), a non-perturbative technique originally developed as the exact solution of a interacting electron problem on an infinite dimensional lattice $[10,11]$. In its original formulation, DMFT solves a general tight-binding problem in the presence of local interactions by mapping it onto an effective local model. The latter is embedded in a quantum medium, whose properties are determined self-consistently.

The results for the optical absorption of small lattice polarons are presented in Section 2. These are generalized in Section 3 to include an additional coupling with the magnetic background. The main findings are briefly summarized in Section 4 .

\section{Optical conductivity for Holstein polarons}

As a paradigmatic model for the study of for small lattice polarons we choose the the Holstein molecular crystal model whose Hamiltonian reads

$$
\begin{aligned}
H & =\omega_{0} \sum_{i} a_{i}^{+} a_{i}-g \sum_{i, \sigma} c_{i}^{+} c_{i}\left(a_{i}^{+}+a_{i}\right) \\
& -t \sum_{i, j}\left(c_{i}^{+} c_{j}+\text { H.c. }\right) .
\end{aligned}
$$

where tight-binding electrons $\left(c_{i}, c_{i}^{\dagger}\right)$ with hopping amplitude $t$ are coupled locally to Einstein bosons $\left(a_{i}, a_{i}^{\dagger}\right)$ with energy $\omega_{0}$. We choose as dimensionless parameters of the model the e-ph coupling constant $\lambda=g^{2} / \omega_{0} D$, where $D$ is the half-bandwidth of our lattice, and the adiabatic ratio $\gamma=\omega_{0} / D$.

The single electron case can be solved semianalytically within DMFT at any temperature [9]. The solution is given in terms of the local Green function $G(\omega)$ [or equivalently a local self-energy $\Sigma(\omega)]$ in the form of a continued fraction expansion. $[12,9]$ The self energy enters in the single particle spectral function $\rho(\epsilon, \nu)=-\frac{1}{\pi} \operatorname{Im} \frac{1}{\nu-\Sigma(\nu)-\epsilon}$.

Its integral over the band dispersion $\epsilon$ equals the local Green's function $G(\omega)$, which provides the selfconsistency equation that closes the DMFT loop.

The conductivity at finite frequency is related to the current-current correlation function through the appropriate Kubo formula. In DMFT, due to the absence of vertex corrections, $[13,14]$ the optical conductivity per particle can be expressed in the low density limit as

$$
\begin{aligned}
\sigma(\omega)= & \frac{\zeta \pi}{\omega}\left(1-e^{-\beta \omega}\right) \int d \epsilon N(\epsilon) \phi(\epsilon) \\
& \times \int d \nu e^{-\beta \nu} \rho^{\mathrm{w}}(\epsilon, \nu) \rho(\epsilon, \nu+\omega)
\end{aligned}
$$

where $\beta=1 / T$ is the inverse temperature, and the constant $\zeta=e^{2} a^{2} / \hbar v$, that we shall omit in the following, carries the appropriate dimensions of conductivity ( $a$ being the lattice spacing, $v$ the volume of the unit cell). In Eq. (3)

$\rho^{\mathrm{w}}(\epsilon, \omega)=\frac{\mathrm{e}^{-\beta \omega} \rho(\epsilon, \omega)}{\int d \epsilon N(\epsilon) \int d \nu \mathrm{e}^{-\beta \nu} \rho(\epsilon, \nu)}$,

defines the "weighted spectral function", which carries information on thermally excited states.

The density of states $N(\epsilon)$ of the unperturbed lattice is assumed semi-elliptical of half bandwidth $D$, namely $N(\epsilon)=\left(2 / \pi D^{2}\right) \sqrt{D^{2}-\epsilon^{2}}$. In Eq. (3), $\phi(\epsilon)=\left(D^{2}-\epsilon^{2}\right) / 3$ is the current vertex function [15-17]. With this choice for $N(\epsilon)$ and $\phi(\epsilon)$, the $\epsilon$ integral can be performed analytically, leaving only one integral to be computed numerically [15].

Let us focus on the adiabatic regime $\gamma \ll 1$, where the polaron crossover is very sharp (it becomes a true localization transition at $\gamma=0$ ). [18] A key parameter which controls the optical properties in this regime is the variance of the phonon quantum fluctuations $s=\lambda D \omega_{0} \operatorname{coth} \omega_{0} / 2 T$. [19] In the strong coupling regime, the photoexcitation of the electron is much faster than the lattice dynamics, which is virtually frozen during the absorption process. Since the lattice energy cannot be relaxed, the dominant optical transition corresponds to the difference in electronic energy between the initial and final states (Franck-Condon principle) which, in the Holstein model, equals 
twice the ground state energy $E_{P}=\lambda D$. The shape of the optical absorption will depend on the ratio between the width of the non-interacting band $\sim D$, and the variance $s$ of the phonon field.

When $s \gg D$, i.e. when the phonon induced broadening of electronic levels is much larger than the electronic dispersion, the absorption by localized polarons takes the form of a skewed Gaussian peak centered at $\omega_{\max }=2 E_{P}[20,21]$

$\sigma(\omega)=\frac{\pi}{\omega} \frac{D^{2}}{4} \frac{1-e^{-\omega / T}}{\sqrt{4 \pi s^{2}}} \exp \left[-\frac{\left(\omega-2 E_{P}\right)^{2}}{4 s^{2}}\right]$.

In the opposite limit $s \ll D$, the lineshape is dominated by the electronic dispersion. The absorption is due to transitions from a polaronic state whose electronic energy is $\simeq-2 E_{P}$ to the continuum of free-electron states $[22,23]$. From Eq. (3) we get in this limit [15]

$$
\begin{aligned}
\sigma(\omega)= & \frac{4 \pi E_{P}^{2}}{\omega^{2}} \frac{1-e^{-\omega / T}}{\omega} \\
& \times \phi\left(\omega-2 E_{P}\right) N\left(\omega-2 E_{P}\right) .
\end{aligned}
$$

We see that in this case the absorption of photons occurs as a threshold phenomenon above $2 E_{P}-$ $D$ and vanishes above $2 E_{P}+D$. The lineshape strongly differs from Eq. (5) and is rather similar to the weak coupling case (the absorption vanishing as a power law at the edges). For the above semielliptical DOS, which can be taken as representative of a three-dimensional lattice, we find that the absorption maximum $\omega_{\max }=2 E_{P}-D^{2} / 2 E_{P}$ is shifted to lower frequencies compared to the usual estimate $2 E_{P}$. This softening is entirely due to finite bandwidth effects. Note that Eq.(6) is valid at all temperatures below the polaron dissociation temperature $T \sim E_{P}$. In particular, contrary to Eq. (5), nothing happens here at temperatures $T \sim$ $\omega_{0} / 2$, provided that the condition $s \ll D$ is not violated (in other words, a temperature independent sub-threshold absorption is not necessarily in contradiction with polaron formation [24]).

Figure 1 shows the DMFT results at $\gamma=0.1$ and $\lambda=1.1$. This value of the coupling strength lies in the polaronic regime, in a region where the electronic dispersion and the phonon-induced broadening are comparable $(s / D=0.33)$, so that none of Eqs. (5) and (6) is expected to hold. DMFT results

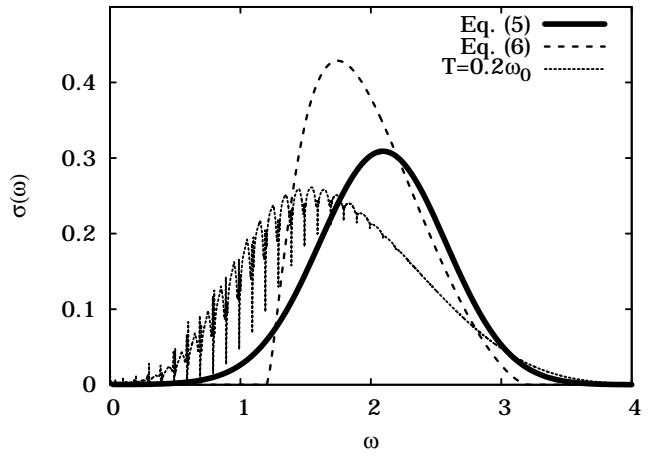

Fig. 1. Optical conductivity for $\lambda=1.1, \gamma=0.1$ and $T / \omega_{0}=0.2$ (dashed lines) compared with $T=0$ predictions of Eq. (5) (full line) and Eq. (6) (bold dashed line).

shows a qualitative difference between low $(\omega<$ $\left.2 E_{P}\right)$ and high $\left(\omega>2 E_{P}\right)$ frequency regions: characteristic phonon resonances are evident at low frequencies, that merge in a broad continuum at higher frequencies $[9,15]$. This behavior, obtained in DMFT, cannot be reproduced by the usual approximate formulas, which either predict a smooth continuum or a multipeaked spectrum depending on their range of validity.[21]

Concerning the overall shape of the optical conductivity, the DMFT spectrum at $s / D=0.33$ is intermediate between the two asymptotic formulas above. It is softer, broader and more asymmetric than Eq. (5) (full line). In fact, the position of the absorption maximum agrees better with Eq. (6) (dashed line). However, the peak height is much reduced compared to Eq. (6) and the absorption edge is completely washed out by phonon fluctuations.

Our results show that detectable deviations from the commonly used Eq. (5) arise as soon as the noninteracting bandwidth is larger than the broadening $s$, a condition that is often realized in real systems. For example, taking typical values $\omega_{0} \simeq$ $0.01-0.05 \mathrm{eV}$ and $E_{p} \simeq 0.1-0.5 \mathrm{eV}$ yields a zero temperature broadening $s \simeq 0.03-0.16 \mathrm{eV}$, in which case electron bandwidths of few tenths of $m e V$ are already sufficient to invalidate the standard Gaussian lineshape Eq. (5). 


\section{Optical Conductivity for Holstein-t- $J$ polarons}

We consider the case of a single hole in an antiferromagnetic (AF) background, interacting with local Holstein phonons. Using the linear spin-wave approximation[25] and neglecting terms that vanish in the limit of large coordination number $z \gg$ 1, we can write the Hamiltonian as:[26]

$$
\begin{aligned}
H & =\frac{t}{2 \sqrt{z}} \sum_{\langle i j\rangle}\left(h_{j}^{\dagger} h_{i} a_{j}+\text { h.c. }\right)+\frac{\tilde{J}}{2} \sum_{i} a_{i}^{\dagger} a_{i}+ \\
& +g \sum_{i} h_{i}^{\dagger} h_{i}\left(b_{i}+b_{i}^{\dagger}\right)+\omega_{0} \sum_{i} b_{i}^{\dagger} b_{i} .
\end{aligned}
$$

The first term of Eq. (7) describes the hopping of one hole $h$ in the antiferromagnetic background. Each hop is accompanied by the creation (destruction) of a spin defect $a$ which breaks (restores) $z$ magnetic bonds with individual energy $\tilde{J} / 2 z=$ $J(1-2 x) / 2 z \cdot x=\left\langle a^{\dagger} a\right\rangle$ is the density of spin defects, which is finite at nonzero temperature. The strength of the local e-ph interaction which couples the hole density to the local phonons is now defined as $\lambda=g^{2} / \omega_{0} t$, and the adiabatic ratio is $\gamma=\omega_{0} / t$. The density of spin defects can be obtained from the magnetization $m=1-2 x$ via the Curie-Weiss equation: $m=\tanh (\beta J m / 4)$ which defines a Néel temperature $T_{\mathrm{N}}=J / 4$.

An exact solution for the thermodynamical and the one-particle spectral properties of this model can be derived as a generalization of the procedure obtained in Ref. [26] at $T=0$, and is again expressed in terms of a continued fraction [27]. Although the formal scheme is similar to the single electron solution of the Holstein model, [9] the DMFT solution of the model Eq. (7) gives a purely local Green's function: the holes are localized at $T=0$ and can move only due to thermal fluctuations of the underlying AF background $[26,28,27]$. In fact the DMFT procedure enforces a classical treatment of the magnetic background, therefore neglecting coherent hole-propagation, which is related to the spin-flip fluctuations. As a consequence, no Drude peak can be observed in the optical conductivity. On the other hand, the incoherent contributions of $\sigma(\omega)$ are mainly dom-

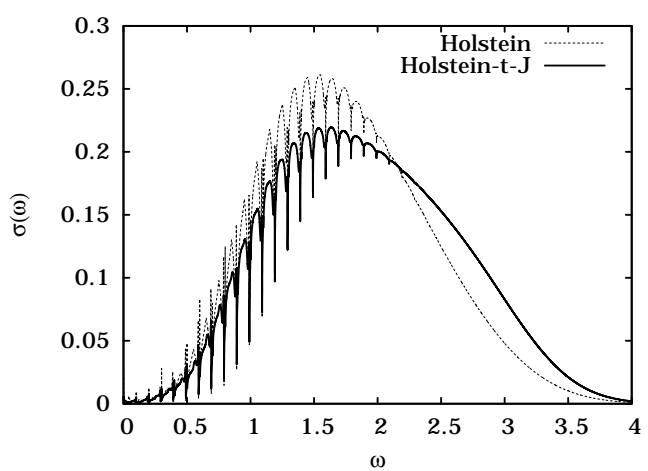

Fig. 2. Comparison between the optical conductivity in the Holstein model and in the paramagnetic $(J=0)$ Holstein-tJ model, at $\lambda=1.1, \gamma=0.1$ and $T / \omega_{0}=0.2$. The full line has been scaled by a factor 4 to compensate for the prefactor $\int N(\epsilon) \phi(\epsilon)=t^{2} / 4$ in the Holstein case.

inated by local properties, such as the local e-ph scattering and spin-string excitations within the magnetic polaron, which are well captured by this approach.[26]

The optical conductivity per hole is expressed as [27]

$\sigma(\omega)=\frac{t^{2} \pi\left(1-\mathrm{e}^{-\beta \omega}\right)}{4 \omega} \int d \Omega \bar{\rho}^{\mathrm{w}}(\Omega)$

$\times[x \bar{\rho}(\omega+\Omega+\tilde{J} / 2)+(1-x) \bar{\rho}(\omega+\Omega-\tilde{J} / 2)]$.

In Eq. (8) $\bar{\rho}(\omega)$ and $\bar{\rho}^{\mathrm{w}}(\omega)$ are respectively the local spectral function and the local weighted spectral function for a single hole localized around a spindefect-free site. The effect of the current vertex function as well as free band dispersion in Eq. (3) is replaced here by the $t^{2}$ prefactor. Note that the interaction with the AF background enters in Eq. (8) explicitly via the effective coupling $\tilde{J}$ and also implicitly via the self-consistent $\bar{\rho}$. In the paramagnetic case, where $\tilde{J}=$ and $x=1 / 2$, the Holstein$t-J$ Eq. (8) does not reduce to pure Holstein result Eq. (3) due to the aforementioned absence of coherent propagation. This is illustrated in Fig. 2, where we have compared the results of both models for a given value of the el-ph parameters, $\lambda=1.1$ and $\gamma=0.1$. The optical lineshape in the paramagnetic Holstein- $t$ - $J$ model (full line) is more asymmetric, i.e. surprisingly more similar to Eq. (6) than to Eq. (5). 
We now examine the effects of the magnetic exchange $J$ on the polaronic optical conductivity in the Holstein- $t-J$ model. We focus on the adiabatic regime, fixing the ratio $\gamma=0.1$ which is a value is qualitatively representative of the cuprates. This regime is also the most interesting one from the theoretical point of view, since in this case the interplay between lattice and spin degrees of freedom has its most dramatic effects.

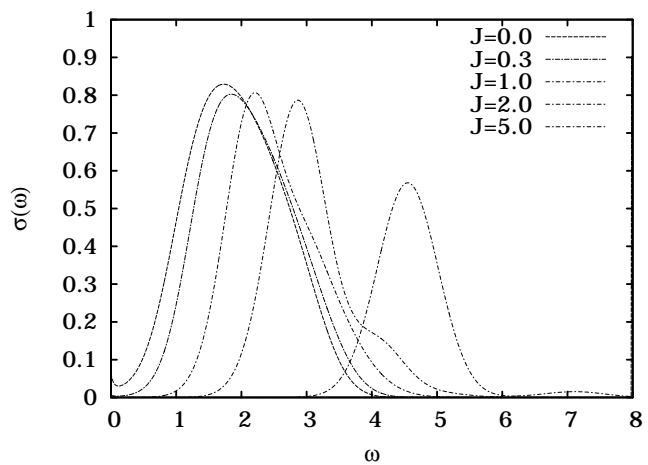

Fig. 3. Optical conductivity at $\lambda=1.1, \gamma=0.1$ and $T / \omega_{0}=0.2$, for different values of $J$.

Fig. 3 shows the evolution of the optical absorption at fixed $\lambda=1.1$ and temperature $T / \omega_{0}=0.2$, upon varying the exchange integral $J$. All curves are calculated using the strong coupling formulation presented in Ref. [27], which is appropriate at such high values of $\lambda$ (only the fine features on the scale of the phonon frequency are lost, resulting in much smoother curves compared to the full DMFT result). The main effect of increasing $J$ is a progressive shift of the spectral weight towards higher frequencies, by a quantity proportional to $J$. This mainly originates from the explicit shift $\sim \tilde{J} / 2$ of the spectral functions in Eq. (8). In addition, the lineshape also changes: magnetic transitions inside the polaron become visible at large $J$. This is already seen at $J=1$ and $J=2$, where the main absorption peak becomes much sharper than at $J=0$, while a second shoulder starts to emerge on the high frequency side. Eventually, at sufficiently strong $J / t \gg \lambda$, several magnon peaks are resolved, each one being dressed by its own lattice cloud (which is responsible for the Gaussian shape of the individual peaks).

\section{Conclusions}

In this work we have compared the optical conductivity obtained by DMFT in two cases: i) small polarons, resulting from a local electron-lattice interaction; ii) small lattice/magnetic polarons, where in addition to the lattice, the carrier is coupled to an AF background.

In the case of lattice polarons, we have emphasized the role of the finite electronic bandwidth, which modifies the optical conductivity from the usual lineshapes. Such effects can be effectively studied using DMFT, because the kinetic and interaction terms are treated on the same footing. In the strong coupling adiabatic regime, the key parameter turns out to be the ratio $s / D$ between the energy spread of the phonon fluctuations and the electron bandwidth. Upon reducing $s / D$, the optical conductivity is found to change from Reik's textbook Gaussian lineshape to a broader absorption band with sharp absorption edges.

In the second case, we have shown that polaronic features are enhanced by the AF correlations. Upon increasing the exchange integral $J$, the absorption band shifts to higher frequencies, testifying the increase in binding energy due to the localization effect of the magnetic environment. When the magnetic scale $J$ becomes larger than the lattice energy scale $\lambda t$, the absorption spectrum eventually separates into several magnon peaks, each one with a Gaussian lineshape, reflecting the attached multiphonon cloud. We emphasize that the electron-lattice interaction is essential in order to have polaronic features in the optical absorption (such as a broad absorption band with a pseudogap at low frequency): the magnetic coupling alone leads to a very different absorption pattern, which consists of a series of sharp magnon peaks [28], with a rapid decay at high frequency.

\section{References}

[1] T. Egami in "Polarons in Bulk and System with Reduced Dimensionality" proc. of Intnl. School of Phys. ed. by G. Iadonisi and J. Ranninger IOS Press (2006)

[2] T. Holstein Ann.Phys. 8, 325 (1959), ibid. 343 (1959). 
[3] S. Fratini and S. Ciuchi, Phys. Rev. Lett. 91, 256403 (2003)

[4] P. Calvani in "Polarons in Bulk and System with Reduced Dimensionality" proc. of Intnl. School of Phys. ed. by G. Iadonisi and J. Ranninger IOS Press (2006)

[5] M.A. Kastner, R.J. Birgeneau, G. Shirane, and Y. Endoh, Rev. Mod. Phys. 70, 897 (1998).

[6] Fehske H., Alvermann A. in "Polarons in Bulk and System with Reduced Dimensionality" proc. of Intnl. School of Phys. ed. by G. Iadonisi and J. Ranninger IOS Press (2006)

[7] S. Fratini and F. de Pasquale, S. Ciuchi Phys. Rev. B 63, 153101 (2001)

[8] A. Lanzara, N. L. Saini, M. Brunelli, F. Natali, A. Bianconi, P. G. Radaelli, and S.-W. Cheong Phys. Rev. Lett. 81, 878 (1998)

[9] S. Ciuchi, F. de Pasquale, S. Fratini and D. Feinberg, Phys. Rev. B 56, 4494 (1997)

[10] Brandt U. Mielsch C. Z. Phys. B 75, 365 (1989)

[11] Muller-Hartmann E. Z.Phys. B 74, 507 (1989)

[12] H. Sumi, J. Phys. Soc. Jpn. 36, 770 (1974)

[13] A. Khurana Phys. Rev. Lett. 64, 1990 (1990).

[14] A.Georges, et al., Rev. Mod. Phys. 68, 13 (1996) and references cited therein.

[15] S. Fratini S. Ciuchi Phys. Rev. B 74, 075101 (2006)

[16] W. Chung and J.K. Freericks, Phys. Rev. B 57, 11955 (1998)

[17] A. Chattopadhyay, A.J. Millis, S.Das Sarma, Phys. Rev. 61, 10738 (2000)

[18] M. Capone, S. Ciuchi, and C. Grimaldi Europhys. Lett. 42, 523 (1998)

[19] S. Fratini and S. Ciuchi, Phys. Rev. B 72, 235107 (2005)

[20] D. M. Eagles, Phys. Rev. 130, 1381 (1963); M. I. Klinger, Phys. Letters 7, 102 (1963); V. N. Bogomolov, E. K. Kudinov, D. N. Mirlin and Yu. A.Firsov, Fiz. Tverd. Tela 9, 2077 (1967) [Sov. Phys. Solid State 9, 1630 (1968)]

[21] H. G. Reik. Sol. St. Comm 1, 102 (1963); H. G. Reik and D. Heese, J. Phys. Chem. Solids 28, 581 (1967); H. G. Reik in "Polarons in Ionic Crystals and Polar Semiconductors", ed. J.Devreese, NorthHolland, Amsterdam (1972)

[22] Yu. A. Firsov, Fiz. Tverd. Tela 10, 1950 (1968) [Sov. Phys. Solid State 10, 1537 (1969)]

[23] A. S. Alexandrov, V. V. Kabanov and D. K. Ray, Physica C 224, 247 (1994)

[24] C. Presura et al., Phys. Rev. Lett. 90, 026402 (2003)
[25] G. Martínez and P. Horsch, Phys. Rev. B 44, 317 (1991).

[26] E. Cappelluti and S. Ciuchi, Phys. Rev. B 66, 165102 (2002).

[27] E. Cappelluti, S. Ciuchi, S. Fratini, preprint arXiv: 0705.1944

[28] M.P.H. Stumpf and D.E. Logan, Eur. Phys. J. B, 8, 377 (1999) 